\title{
Preliminary Design of a Manned Nuclear Electric Propulsion Vehicle Using Genetic Algorithms
}

\author{
Ryan W. Irwin ${ }^{1}$ and Michael L. Tinker ${ }^{2}$ \\ 'School of Aeronautics and Astronautics, Purdue University, West Lafayette, IN 47907 \\ ${ }^{2}$ Spacecraft and Vehicle Systems Department, NASA Marshall Space Flight Center, Huntsville, AL 35812 \\ 256-544-5973, mike.tinker@nasa.gov'
}

\begin{abstract}
Nuclear electric propulsion (NEP) vehicles will be needed for future manned missions to Mars and beyond. Candidate designs must be identified for further detailed design from a large array of possibilities. Genetic algorithms have proven their utility in conceptual design studies by effectively searching a large design space to pinpoint unique optimal designs. This research combined analysis codes for NEP subsystems with a genetic algorithm. The use of penalty functions with scaling ratios was investigated to increase computational efficiency. Also, the selection of design variables for optimization was considered to reduce computation time without losing beneficial design search space. Finally, trend analysis of a reference mission to the asteroids yielded a group of candidate designs for further analysis.
\end{abstract}

\section{INTRODUCTION}

The announcement of the Vision for Space Exploration has resulted in renewed focus on human exploration of Mars and other distant locations. Nuclear electric propulsion (NEP) has been identified as a front runner for these missions that necessitate short trip times to minimize crew cosmic radiation exposure. While numerous manned chemical propulsion vehicles have been designed in the past, there is considerably less experience for nuclear electric propulsion vehicles. In addition to traditional aerospace knowledge areas, competent NEP design requires knowledge of nuclear reactors and electric thrusters as well as conversion, management, and distribution of thermal and electrical energy. A thorough search of the design possibilities for these vehicles is needed for a proper assessment of mission viability and identification of feasible low-mass candidates for future designs. Genetic algorithms have demonstrated an ability to find global optima over large search spaces such as this. Genetic algorithms are suited for preliminary design problems because they can be used with both continuous and discrete design variables and can find areas of optimal designs for later detailed design analysis. This research aims at finding candidate nuclear electric vehicle designs for a manned mission to the asteroid belt.

\section{NUCLEAR ELECTRIC PROPULSION}

Nuclear electric propulsion consists of using a nuclear energy source (such as a fission reactor) to supply high power levels for electric thrusters. A basic NEP vehicle system layout is given in Fig. 1. A power conversion system is needed to convert the thermal power output from the reactor into electric power. A manned mission to another planet would require thruster power greater than one megawatt, perhaps as much as 20 megawatts. These high power levels coupled with the small available reactor power conversion efficiencies create large amounts of waste heat that must be dissipated by radiators. Currently, heat pipe radiators are considered the best option because the spacecraft can survive the loss of several heat pipes with only small loss in rejection ability (Smith, 2003).

The presence of radiation from the reactor requires a shadow shield for the crew habitat and other subsystem components. High shield densities drive the need for a large separation distance between the habitat and the reactor, accomplished by a truss. Increasing the separation distance results in thinner shields and smaller shadow angles, 
The points of crossover and mutation are deiermined randomily, and the rate at which they occur can be defined by the user. When the new generation is filled, the process is repeated. Thus, the genetic algorithm uses a random, directed search to find global optima. The genetic operations allow for exploration of a large design space during simultaneous optimization.

For design problems there are often many constraints to consider. Penalty functions are used as a means to penalize the fitness of a solution for constraint violations. If the constraint is satisfied, then the penalty function value is zero. For instance, in the case of NEP vehicle mass optimization, the fitness function should be the sum of the system mass and any penalty functions for constraint violations. Generally, penalty functions are implemented by multiplying a scaling factor with a ratio based on the degree of the constraint violation. Determining an appropriate scaling factor involves some experimentation, as is discussed in later sections of this paper.

\section{NUCLEAR ELECTRIC VEHICLE OPTIMIZATION TOOLSET (NEVOT)}

In order to combine the optimization power of a genetic algorithm with nuclear electric vehicle subsystem analyses, the Nuclear Electric Vehicle Optimization Toolset (NEVOT) was created (Tinker, et al., 2004). NEVOT is a joint effort of Marshall Space Flight Center, Oak Ridge National Laboratories, and the Arnold Engineering Development Center. Figure 2 displays the general organization of NEVOT and its sublevel codes. There are seven analysis modules: Trajectory, Space Reactor Power System (SRPS), Power Management and Distribution (PMAD), Electric Propulsion (EP), Habitat (HAB), Truss, and Configuration (CONFIG). The input and output files for these modules are controlled in the simulation executive. This simulator combines all of the mass and performance data from the subsystem modules to calculate the system mass and assess any penalty functions for constraint violations. It is also the main code that communicates with the genetic algorithm by receiving variables for the design solutions and sending the corresponding fitness value back. The genetic algorithm in Sandia Laboratories' Design Analysis Kit for Optimization and Terascale Applications (DAKOTA) software package was used for the optimization.

The current SRPS code is ALKASYS, a program developed at Oak Ridge National Laboratories for analysis of Rankine-cycle space nuclear power systems. Also, the PMAD code is a program taken from a collaborative effort between Rocketdyne and NASA's Glenn Research Center. The other analysis modules were developed by NEVOT project team members. The modularity of the NEVOT layout easily permits updates to these individual analysis modules. As codes are upgraded for increased accuracy and fidelity, they can be exchanged for previous versions of the codes in the computational architecture. One example of this has been the use of ALKASYS instead of RSMASS. RSMASS is a lower-fidelity reactor code that was used early in the project, but was removed after inspection showed its analysis was unreasonable over the mission power range.

Currently NEVOT allows the GA to work on fourteen design variables: thruster jet power, thruster type (ion, Hall, or MPD), truss length, reactor type (boiling potassium or lithium cooled), condensing temperature, number of turbines, truss member outer diameter, truss member wall thickness, number of truss members, PMAD alternator operating frequency, thruster input voltage, transmission line length, propellant mass, and habitat diameter. Other values pertinent to the codes have been set by engineering judgment. Note that many of the variables are used in more than one subsystem analysis code. Also, the output and input power levels between connected subsystems must be consistent. Due to these dependencies, some subsystem codes must be nun sequentially. Additionally, the configuration module is run last since it uses the geometry of the other subsystems to size the shadow shield angle and diameters.

When a design is passed to the simulator, the subsystem modules are run for analysis of performance. The trajectory module uses the thruster type and jet power to determine a mission trip time. This is later compared to a constraint target mission time chosen for crew health reasons. This trip time also is used in calculating the propellant mass required for the mission based on the jet power and specific impulse of the thrusters. This propellant mass requirement provides a constraint to check against the design propellant mass. The EP code is then run to obtain tank and thruster masses as well as the thrust force and thruster efficiency needed to calculate the PMAD output power. Then PMAD module is run to obtain the mass and efficiency needed to calculate the SRPS output power. At this point, the reactor and power conversion subsystems are analyzed. This analysis yields SRPS and radiator masses as well as the required neutron and gamma shield thicknesses to protect the habitat. Afterwards, the truss module calculates the truss mass and critical buckling force for constraint comparison with the thrust. Also, the 
The penalty ratio is always between zero and one if the design and constraint values are both positive. This ratio must be scaled so that it can impact the fitness function when added to the vehicle mass. If a scale factor is too low, then the fitness function is not significantly affected by constraint violations. The "optimal" design output by the GA may have very low mass but also violate many constraints. On the other hand, if a scale factor is too high the optimization results will be conservative because there will be a large fitness difference between designs that violate constraints and those that do not. The goal is to find a compromise wherein solutions with small constraint violations but low mass are not eliminated immediately. These designs, while not completely feasible, are very close to those designs that meet the constraints at low mass. Thus, their genetic data should be competitive against high-mass designs with all constraints satisfied.

Early in the project it was decided that the design vehicle mass should be used as the penalty scaling factor. Results of the runs showed that the optimal designs were always violating many of the constraints. These designs had such lower mass than some other designs in the same generation that the difference in penalty scaling was too large. Effectively these designs were not assessed as much penalty mass for large constraint violations as those designs with higher mass and small penalty violations. A new scaling factor was needed that would be unbiased within a generation.

A value of $600,000 \mathrm{~kg}$ (a conservative estimate for optimal vehicle mass) was chosen so that conservative designs could be selected to ensure that the mission was possible. When this penalty scaling factor was implemented, the optimal designs often only violated the specific power constraint. It was apparent that a specific power of $4 \mathrm{~kg} / \mathrm{kW}$ was too ambitious for this manned mission with the available analyses. Since the trajectory assumptions of 200,000 $\mathrm{kg}$ vehicle initial mass and $4 \mathrm{~kg} / \mathrm{kW}$ specific power could not be met, the trip time scaling factor was decreased so that the trajectory code would have smaller effect on the optimal designs. This showed that scaling factors for separate constraints could be varied to give priority to one constraint over the other. This allowed the code to optimize for mass and specific power in an attempt to offset the errors in propellant mass calculations by the trajectory code.

\section{Choosing Design Variables for Optimization}

A major challenge in systems design optimization is choosing which parameters to designate as design variables for the code. It is possible to choose every variable on the vehicle, but the resulting computation time would be enormous. Additionally, the purposes of this systems desien study were to identify parameters having the greatest impact on system performance, and to determine appropriate values for the parameters. There are many parameters that are dependent on other parameters and can be calculated based on engineering analysis. Adding dependent parameters to the variable list also adds the necessity for constraint functions. For instance, the critical buckling force of the truss (a result of several design variables) must be checked against the actual delivered thrust of the electric propulsion device (a result of several other design variables). Adding constraint functions forces the genetic algorithm to explore more constraint values and consume greater computational resources. If the designers can choose to design a dependent parameter to its constraint, then they should implement that logic into their analysis codes.

Initially, five design variables and constraints were utilized in addition to those mentioned in the previous sections. These variables were the shield smaller diameter, gamma shield thickness, neutron shield thickness, shadow shield angle, and radiator area. These values were checked against calculated constraints for each. Inspection of the relationships showed that the shield shadow angle and small diameter could be calculated based on vehicle geometries. The other three variables can be designed to the calculated constraints from the SRPS code. Having a thicker shield than is necessary for a certain truss length only adds waste mass to the vehicle. The result of extra radiator area over the waste rejection requirement is the same. By removing these five variables and their five associated constraints, the GA was able to work on fewer variables and constraint functions without loss of quality design space.

Table 1 shows a common occurrence with optimal solutions before the variable selections were changed. For example, the difference in the order of magnitudes of the shield thicknesses can be seen. The radiator is also almost three times as large as required. The extra masses due to the inefficient design of the shield and radiators added up to $120,000 \mathrm{~kg}$, a significant portion of the vehicle total mass. Although long GA nuns were used, the variable values in the optimal designs never converged to their constraints. The shadow shield angle is left out of Table 1 , because 
